\title{
Robust Nonfragile Controllers Design for Fractional Order Large-Scale Uncertain Systems with a Commensurate Order $1<\alpha<2$
}

\author{
Jianyu Lin ${ }^{1,2}$ \\ ${ }^{1}$ Department of Automation, Shanghai Jiao Tong University, Shanghai 200240, China \\ ${ }^{2}$ Department of Communication, Shanghai University of Electric Power, Shanghai 200090, China \\ Correspondence should be addressed to Jianyu Lin; franklinshhai@gmail.com
}

Received 12 July 2014; Accepted 10 September 2014

Academic Editor: Dan Ye

Copyright (C) 2015 Jianyu Lin. This is an open access article distributed under the Creative Commons Attribution License, which permits unrestricted use, distribution, and reproduction in any medium, provided the original work is properly cited.

\begin{abstract}
The paper concerns the problem of stabilization of large-scale fractional order uncertain systems with a commensurate order $1<\alpha<2$ under controller gain uncertainties. The uncertainties are of norm-bounded type. Based on the stability criterion of fractional order system, sufficient conditions on the decentralized stabilization of fractional order large-scale uncertain systems in both cases of additive and multiplicative gain perturbations are established by using the complex Lyapunov inequality. Moreover, the decentralized nonfragile controllers are designed. Finally, some numerical examples are given to validate the proposed method.
\end{abstract}

\section{Introduction}

In the past decades, a great deal of attention has been paid to the stability and stabilization of large-scale systems [1-7]. This is due to the fact that there exist a large number of large-scale interconnected dynamical systems in many practical physical systems, such as process control systems, computer communication networks, transportation systems, and economic systems. Meanwhile, nonfragile controllers have been nominated by resilient and the fragility of the PID controllers has been analyzed in [8]. The controller gain perturbations can commonly be modeled as uncertain gains which are dependent on uncertain parameters in the literature $[9,10]$. The robust nonfragile control problem for uncertain integer order large-scale system has been studied [11-13]. In recent years, the nonfragile control problem has been an attractive topic in theory analysis and practical implement, because of perturbations often appearing in the controller gain, which may result in either the actuator degradations or the requirements for readjustment of controller gains. The problem of reliable dissipative control within nonfragile control framework has been investigated in [14, 15]. The nonfragile control idea is how to design a feedback control that will be insensitive to perturbations in gains of feedback control. The robust resilient stabilization problem is to design a nonfragile state feedback controller such that the uncertain fractional order large-scale interconnected closedloop system with a commensurate order $1<\alpha<2$ is robustly stable for all admissible parameter uncertainties.

On the other hand, pioneering works in stability analysis and stabilization of fractional order control systems can be found in [16-20]. The robust stability of fractional order interval systems has been investigated in [21, 22]. It is well known that Matignon's stability theorem [16] is the basis for stability analysis of the fractional order system by checking the location of eigenvalues in the complex plane. Matignon's theorem is in fact the pioneering works of stability analysis of the fractional order system. Based on Matignon's theorem, the stability criteria of fractional order systems have been proposed in both cases of $1<\alpha<2$ and $0<\alpha<1$ in $[23,24]$. The necessary and sufficient LMI conditions for stability analysis of a commensurate fractional order system have been established in $[23,24]$, in which complex Lyapunov inequality holds. However, very few studies provide LMI conditions for the stability analysis of the fractional order large-scale interconnected system in the literature. Our study is mainly motivated by the works $[23,24]$. The important feature is that the proposed method can be implemented to 
the fractional order large-scale interconnected system. The objective of the paper is to design a nonfragile controller which is robust to system uncertainties and resilient to controller gain variations for the fractional order large-scale interconnected systems with a commensurate order $1<\alpha<$ 2. Here, it should be also pointed out that [25] only focuses on the case of a fractional order $0<\alpha<1$. This paper is organized as follows. Some preliminaries and the problem statement are given in Section 2. The main results of the sufficient condition of stabilization of the fractional order system under additive gain perturbations are presented in Section 3. Furthermore, the decentralized stabilization state feedback controller are designed. Meanwhile, the LMI results of the sufficient condition of stabilization of the fractional order system under multiplicative gain perturbations are presented in Section 4. The examples are given in Section 5 to illustrate the effectiveness of our LMI-based results for checking the stabilization of the fractional order large-scale interconnected system. Finally, a brief conclusion is drawn in Section 6.

Notations. Throughout the paper, we denote by $\bar{r}$ the conjugate of the complex number $r$. $j$ denotes the imaginary unit. $I$ denotes the identity matrix with appropriate dimensions. block diag denotes the block diagonal matrix. $R^{n}$ denotes the $n$-dimensional Euclidean space and $R^{n \times m}$ is the set of all $n \times m$ real matrices. $M^{T}$ denotes the transpose of $M$ and $M^{*}$ denotes the Hermitian transpose of $M . \operatorname{Re}()$ and $\operatorname{Im}()$ are corresponding to the real and imaginary parts of the matrix, respectively.

\section{Preliminaries and Problem Formulation}

Let us consider a fractional order large-scale interconnected uncertain system with a commensurate order $1<\alpha<2$ composed of $N$ fractional order subsystems:

$$
\begin{aligned}
\frac{d^{\alpha} x_{i}(t)}{d t^{\alpha}}= & {\left[A_{i i}+\Delta A_{i i}\right] x_{i}(t) } \\
& +\sum_{j=1, j \neq i}^{N}\left[A_{i j}+\Delta A_{i j}\right] x_{j}(t)+B_{i} u_{i}(t),
\end{aligned}
$$

where $\alpha \in R$ is the fractional commensurate order, $i, j=$ $1,2, \ldots N$, and $x_{i}(t) \in R^{n_{i}}$ and $u_{i}(t) \in R^{m_{i}}$ are the state and input of the $i$ th fractional order subsystem, respectively. Assume that the nominal systems $A_{i i} \in R^{n_{i} \times n_{i}}, A_{i j} \in R^{n_{i} \times n_{i}}$, and $B_{i} \in R^{n_{i} \times m_{i}}$ are constant and of appropriate dimensions and the pair $\left(A_{i i}, B_{i}\right)$ is controllable. The fractional order subsystems interact with each other through the interconnections $\sum_{j=1, j \neq i}^{N} A_{i j} x_{j}(t)$. The main objective of the note is to find the decentralized local state feedback control law of the following form:

$$
u_{i}(t)=\left(K_{i}+\Delta K_{i}\right) x_{i}(t), \quad i=1,2, \ldots, N,
$$

such that the resulting fractional order closed-loop system is asymptotically stable, where $K_{i} \in R^{m_{i} \times n_{i}}$ is the state feedback gain matrix to be designed and $\Delta K_{i}=D_{b i} F_{b i} E_{b i}$ and
$\Delta K_{i}=D_{d i} F_{d i} E_{d i} K_{i}$ represent the additive and multiplicative gain perturbations, respectively. In this note, the uncertainty is bounded as follows. The parameter uncertainties considered here are norm-bounded and are of the forms $\Delta A_{i i}=$ $D_{a i i} F_{a i i} E_{a i i}, F_{a i i}^{T} F_{a i i} \leq I ; \Delta A_{i j}=D_{a i j} F_{a i j} E_{a i j}, F_{a i j}^{T} F_{a i j} \leq I$; $\Delta K_{i}=D_{b i} F_{b i} E_{b i}, F_{b i}^{T} F_{b i} \leq I ; \Delta K_{i}=D_{d i} F_{d i} E_{d i} K_{i}, F_{d i}^{T} F_{d i} \leq I$, where the elements are Lebesgue measurable and $D_{a i i}, D_{a i j}$, $D_{b i}, D_{d i}, E_{a i i}, E_{a i j}, E_{b i}$, and $E_{d i}$ are known real matrices of appropriate dimensions which characterize the structure of the uncertainty. The overall system is described by the composite fractional order large-scale state equations

$$
\frac{d^{\alpha} x(t)}{d t^{\alpha}}=(A+\Delta A) x(t)+B(K+\Delta K) x(t),
$$

with the composite matrices $A$ and $K$ having the structure

$$
\begin{gathered}
A=\left[\begin{array}{ccc}
A_{11} & \cdots & A_{1 N} \\
\vdots & \ddots & \vdots \\
A_{N 1} & \cdots & A_{N N}
\end{array}\right], \\
\Delta A=\left[\begin{array}{ccc}
\Delta A_{11} & \cdots & \Delta A_{1 N} \\
\vdots & \ddots & \vdots \\
\Delta A_{N 1} & \cdots & \Delta A_{N N}
\end{array}\right], \\
K=\text { block } \operatorname{diag}\left[K_{1}, K_{2} \cdots K_{N}\right], \\
\Delta K=\text { block } \operatorname{diag}\left[\Delta K_{1}, \Delta K_{2} \cdots \Delta K_{N}\right] .
\end{gathered}
$$

Definition 1 (see [26]). For all nonzero real vectors $X \in R^{n}$, $A \in R^{n \times n}$ is real matrix; if the inequality $X^{T} A X<0$ holds, then $A$ is said to be negative definite matrix.

Definition 2. The fractional order large-scale uncertain system can be stabilized via decentralized state feedback $u_{i}(t)=$ $\left(K_{i}+\Delta K_{i}\right) x_{i}(t)$ if there exists gain matrix $K_{i} \in R^{m_{i} \times n_{i}}$ such that the closed-loop fractional order large-scale uncertain system

$$
\frac{d^{\alpha} x(t)}{d t^{\alpha}}=(A+\Delta A) x(t)+B(K+\Delta K) x(t)
$$

is asymptotically stable.

\section{Nonfragile Controller Design of Fractional Order Large-Scale System with Additive Gain Perturbations}

In this section, the resilient controller synthesis problem is formulated for the fractional order large-scale interconnected system under additive gain perturbations. Sufficient conditions are firstly derived for the decentralized stabilization of fractional order large-scale interconnected system with norm-bounded uncertainties given by (1). Before proceeding further, we will state the following well-known lemmas. We will use the lemmas and theorems to establish sufficient conditions on decentralized stabilization of fractional order large-scale interconnected system with norm-bounded uncertainties under additive gain perturbations.

Lemma 3 (see [27]). For all $C \in R^{n \times k}, A, B \in R^{n \times n}, A \geq B$; then $C^{T} A C \geq C^{T} B C$. 
Lemma 4 (see [27]). For any matrices $X$ and $Y$ with appropriate dimensions and for any $\beta>0$, the following inequality holds:

$$
X^{T} Y+Y^{T} X<\beta X^{T} X+\beta^{-1} Y^{T} Y .
$$

Lemma 5 (see [28]). The fractional order system $d^{\alpha} x(t) / d t^{\alpha}=$ $A x(t)$ with a commensurate order $\alpha$ is asymptotically stable if $|\arg (\operatorname{spec}(A))|>\alpha(\pi / 2)$, where $\alpha$ is the order of fractional order system and $\operatorname{spec}(A)$ is the spectrum of all eigenvalues of A.

Lemma 6 (see [23]). Let $1<\alpha<2$ and $\theta=(\alpha-1)(\pi / 2)$, $r=e^{j \theta}$. The fractional order system $d^{\alpha} x(t) / d t^{\alpha}=A x(t)$ with a commensurate order $1<\alpha<2$ is asymptotically stable if and only if there exist positive definite matrices $P=P^{T} \in R^{n \times n}$, such that

$$
\left[\begin{array}{ll}
\left(A P+P A^{T}\right) \sin \theta & \left(A P-P A^{T}\right) \cos \theta \\
\left(P A^{T}-A P\right) \cos \theta & \left(A P+P A^{T}\right) \sin \theta
\end{array}\right]<0,
$$

or equivalently, $r P A+\bar{r} A^{T} P<0$.

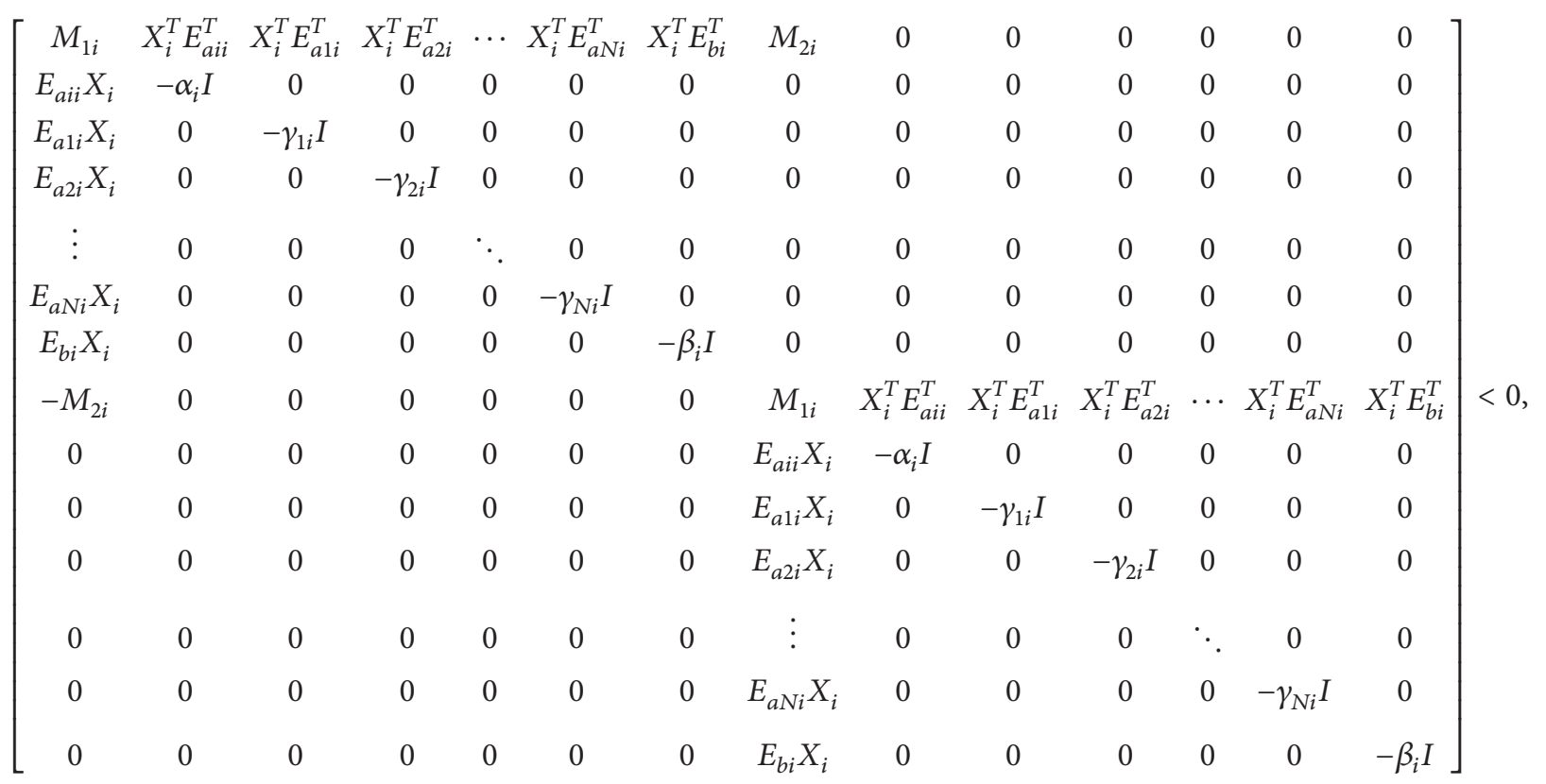

where $M_{i}=r P_{i} A_{i i}+\bar{r} A_{i i}^{T} P_{i}+r P_{i} A_{i j}+\bar{r} A_{i j}^{T} P_{i}+\alpha_{i} D_{a i i} D_{a i i}^{T}+$ $\sum_{j=1, j}^{N} \gamma_{i j} D_{a i j} D_{a i j}^{T}+\beta_{i} B_{i} D_{b i} D_{b i}^{T} B_{i}^{T}+r B_{i} Y_{i}+\bar{r} Y_{i}^{T} B_{i}^{T}, X_{i}=$ $P_{i}^{-1}$, and $M_{1 i}$ and $M_{2 i}$ are the real part and imaginary part of matrices $M_{i}$, respectively. Moreover, the stabilization decentralized state-feedback gain matrix can be calculated as follows: $K_{i}=Y_{i} P_{i}$.

Proof. Under decentralized state-feedback control law (2), the closed-loop fractional order large-scale interconnected
Proof. The idea is mainly based on the geometric analysis of a fractional system stability domain. Based on Lemma 5, the stability domain for a fractional order $1<\alpha<2$ is convex. By using Linear Matrix Inequalities (LMI) approach, it is obtained as the above LMI. Therefore, it is equivalent to $r P A+\bar{r} A^{T} P<0$.

Lemma 7. A complex Hermitian matrix $M$ satisfies $M<0$ if and only if the following real LMI inequality holds:

$$
\left[\begin{array}{cc}
\operatorname{Re}(M) & \operatorname{Im}(M) \\
-\operatorname{Im}(M) & \operatorname{Re}(M)
\end{array}\right]<0 .
$$

Under commensurate order hypothesis, our finding is summarized in the following theorem.

Theorem 8. Consider the fractional order large-scale intercon$e^{j(\alpha-1)(\pi / 2)}$. The fractional order large-scale uncertain system can be stabilized via decentralized state feedback $u_{i}(t)=\left(K_{i}+\right.$ $\left.\Delta K_{i}\right) x_{i}(t)$ if there exist positive-definite block diagonal matrices $P_{i}=$ block $\operatorname{diag}\left[P_{1}, P_{2} \ldots, P_{N}\right]$, matrix $Y_{i}$, and positive real scalar constants $\alpha_{i}, \gamma_{i j}, \beta_{i}, i, j=1,2, \ldots, N$, such that the following matrix inequalities hold: nected system (1) with a commensurate order $1<\alpha<2$. Let $r=$ (1) 
$r P A+\bar{r} A^{T} P<0$. According to Definition 1, the sufficient condition on the stabilization of fractional order largescale interconnected system satisfies the following quadratic matrix inequality:

$$
\begin{aligned}
& \zeta^{T}\{r P[(A+\Delta A)+B(K+\Delta K)] \\
& \left.+\bar{r}[(A+\Delta A)+B(K+\Delta K)]^{T} P\right\} \zeta<0, \\
& \sum_{i=1}^{N}\left\{\zeta _ { i } ^ { * } \left[r P_{i} A_{i i}+\bar{r} A_{i i}^{T} P_{i}+r P_{i} \Delta A_{i i}\right.\right. \\
& +\bar{r} \Delta A_{i i}^{T} P_{i}+r P_{i} B_{i} K_{i}+\bar{r} K_{i}^{T} B_{i}^{T} P_{i} \\
& \left.+r P_{i} B_{i} \Delta K_{i}+\bar{r} P_{i} \Delta K_{i}^{T} B_{i}^{T}\right] \zeta_{i} \\
& \left.+2 r \zeta_{i}^{*}\left[\sum_{j=1, j \neq i}^{N}\left(A_{i j}+\Delta A_{i j}\right)\right] \zeta_{j}\right\}<0 .
\end{aligned}
$$

Consequently, the sufficient condition on the decentralized stabilization of fractional order large-scale interconnected system is that quadratic matrix inequality (11) holds.

Based on Lemmas 3 and 4, by means of enlarging the inequality, it yields

$$
r P_{i} \Delta A_{i i}+\bar{r} \Delta A_{i i}^{T} P_{i} \leq \alpha_{i} P_{i} D_{a i i} D_{a i i}^{T} P_{i}+\alpha_{i}^{-1} E_{a i i}^{T} E_{a i i} .
$$

Meanwhile, based on Lemmas 3 and 4, by means of enlarging the inequality we have

$$
\begin{gathered}
r P_{i} B_{i} \Delta K_{i}+\bar{r} \Delta K_{i}^{T} B_{i}^{T} P_{i} \leq \beta_{i} P_{i} B_{i} D_{b i} D_{b i}^{T} B_{i}^{T} P_{i}+\beta_{i}^{-1} E_{b i}^{T} E_{b i}, \\
\sum_{i=1}^{N} \sum_{j=1, j \neq i}^{N} \zeta_{i}^{*}\left[r P_{i} \Delta A_{i j}+\bar{r} \Delta A_{i j}^{T} P_{i}\right] \zeta_{j} \\
\leq \sum_{i=1}^{N} \sum_{j=1, j \neq i}^{N} \zeta_{i}^{*} \gamma_{i j} P_{i} D_{a i j} D_{a i j}^{T} P_{i} \zeta_{i} \\
+\sum_{j=1, j \neq i}^{N} \sum_{i=1}^{N} \zeta_{j}^{*} \gamma_{i j}^{-1} E_{a i j}^{T} E_{a i j} \zeta_{j} \\
\leq \sum_{i=1}^{N} \sum_{j=1, j \neq i}^{N} \zeta_{i}^{*} \gamma_{i j} P_{i} D_{a i j} D_{a i j}^{T} P_{i} \zeta_{i} \\
+\sum_{j=1, j \neq i}^{N} \sum_{i=1}^{N} \zeta_{i}^{*} \gamma_{j i}^{-1} E_{a j i}^{T} E_{a j i} \zeta_{i} .
\end{gathered}
$$

Substituting (11), (12), and (13) into (12) results in the following quadratic matrix inequality:

$$
\begin{aligned}
& \sum_{i=1}^{N}\left\{\zeta _ { i } ^ { * } \left[r P_{i} A_{i i}+\bar{r} A_{i i}^{T} P_{i}+r P_{i} A_{i j}+\bar{r} A_{i j}^{T} P_{i}\right.\right. \\
& +r P_{i} B_{i} K_{i}+\bar{r} K_{i}^{T} B_{i}^{T} P_{i} \\
& +\alpha_{i} P_{i} D_{a i i} D_{a i i}^{T} P_{i}+\sum_{j=1, j \neq i}^{N} \gamma_{i j} P_{i} D_{a i j} D_{a i j}^{T} P_{i} \\
& +\alpha_{i}^{-1} E_{a i i}^{T} E_{a i i}+\sum_{j=1, j \neq i}^{N} \gamma_{i j}^{-1} E_{a i j}^{T} E_{a i j} \\
& \left.\left.+\beta_{i}^{-1} E_{b i}^{T} E_{b i}+\beta_{i} P_{i} B_{i} D_{b i} D_{b i}^{T} B_{i}^{T} P_{i}\right] \zeta_{i}\right\}<0, \\
& \sum_{i=1}^{N}\left\{r P_{i} A_{i i}+\bar{r} A_{i i}^{T} P_{i}+r P_{i} A_{i j}+\bar{r} A_{i j}^{T} P_{i}\right. \\
& +r P_{i} B_{i} K_{i}+\bar{r} K_{i}^{T} B_{i}^{T} P_{i} \\
& +\alpha_{i} P_{i} D_{a i i} D_{a i i}^{T} P_{i}+P_{i} \sum_{j=1, j \neq i}^{N}\left(\gamma_{i j} D_{a i j} D_{a i j}^{T}\right) P_{i} \\
& +\alpha_{i}^{-1} E_{a i i}^{T} E_{a i i}+\sum_{j=1, j \neq i}^{N} \gamma_{i j}^{-1} E_{a i j}^{T} E_{a i j} \\
& \left.+\beta_{i} P_{i} B_{i} D_{b i} D_{b i}^{T} B_{i}^{T} P_{i}+\beta_{i}^{-1} E_{b i}^{T} E_{b i}\right\}<0 .
\end{aligned}
$$

Let $X_{i}=P_{i}^{-1}$ and $Y_{i}=K_{i} X_{i}$. By premultiplying and postmultiplying $P_{i}^{-1}$ onto (16), one has

$$
\begin{aligned}
& \sum_{i=1}^{N}\left\{r A_{i i} P_{i}^{-1}+\bar{r} P_{i}^{-1} A_{i i}^{T}+r A_{i j} P_{i}^{-1}+\bar{r} P_{i}^{-1} A_{i j}^{T}\right. \\
& +r B_{i} K_{i} P_{i}^{-1}+\bar{r} P_{i}^{-1} K_{i}^{T} B_{i}^{T}+\alpha_{i} D_{a i i} D_{a i i}^{T} \\
& +\sum_{j=1, j \neq i}^{N} \gamma_{i j} D_{a i j} D_{a i j}^{T}+\alpha_{i}^{-1} P_{i}^{-1} E_{a i i}^{T} E_{a i i} P_{i}^{-1} \\
& +\sum_{j=1, j \neq i}^{N} \gamma_{i j}^{-1} P_{i}^{-1} E_{a i j}^{T} E_{a i j} P_{i}^{-1}+\beta_{i} B_{i} D_{b i} D_{b i}^{T} B_{i}^{T} \\
& \left.+\beta_{i}^{-1} P_{i}^{-1} E_{b i}^{T} E_{b i} P_{i}^{-1}\right\} \\
& =\sum_{i=1}^{N}\left\{r A_{i i} X_{i}+\bar{r} X_{i} A_{i i}^{T}+r A_{i j} X_{i}+\bar{r} X_{i} A_{i j}^{T}\right. \\
& +r B_{i} Y_{i}+\bar{r} Y_{i}^{T} B_{i}^{T}+\alpha_{i} D_{a i i} D_{a i i}^{T} \\
& +\sum_{j=1, j \neq i}^{N} \gamma_{i j} D_{a i j} D_{a i j}^{T}+\alpha_{i}^{-1} X_{i} E_{a i i}^{T} E_{a i i} X_{i}
\end{aligned}
$$




$$
\begin{aligned}
& +\sum_{j=1, j \neq i}^{N} \gamma_{i j}^{-1} X_{i} E_{a i j}^{T} E_{a i j} X_{i} \\
& \left.+\beta_{i} B_{i} D_{b i} D_{b i}^{T} B_{i}^{T}+\beta_{i}^{-1} X_{i}^{T} E_{b i}^{T} E_{b i} X_{i}\right\}<0 .
\end{aligned}
$$

If the quadratic matrix inequality holds, then the fractional order large-scale interconnected system is asymptotically stable.

By applying Schur complement, the above matrix inequality is equivalent to the following complex LMI:

$$
\left[\begin{array}{ccccccc}
M_{i} & X_{i}^{T} E_{a i i}^{T} & X_{i}^{T} E_{a 1 i}^{T} & X_{i}^{T} E_{a 2 i}^{T} & \cdots & X_{i}^{T} E_{a N i}^{T} & X_{i}^{T} E_{b i}^{T} \\
E_{a i i} X_{i} & -\alpha_{i} I & 0 & 0 & 0 & 0 & 0 \\
E_{a 1 i} X_{i} & 0 & -\gamma_{1 i} I & 0 & 0 & 0 & 0 \\
E_{a 2 i} X_{i} & 0 & 0 & -\gamma_{2 i} I & 0 & 0 & 0 \\
\vdots & 0 & 0 & 0 & \ddots & 0 & 0 \\
E_{a N i} X_{i} & 0 & 0 & 0 & 0 & -\gamma_{N i} I & 0 \\
E_{b i} X_{i} & 0 & 0 & 0 & 0 & 0 & -\beta_{i} I
\end{array}\right]
$$

$<0$,

(18) where $M_{i}=r P_{i} A_{i i}+\bar{r} A_{i i}^{T} P_{i}+r P_{i} A_{i j}+\bar{r} A_{i j}^{T} P_{i}+\alpha_{i} D_{a i i} D_{a i i}^{T}$ $+\sum_{j=1, j \neq i}^{N} \gamma_{i j} D_{a i j} D_{a i j}^{T}+\beta_{i} B_{i} D_{b i} D_{b i}^{T} B_{i}^{T}+r B_{i} Y_{i}+\bar{r} Y_{i}^{T} B_{i}^{T}$. In practice, the feedback matrix $K_{i}$ has no imaginary part. So let $\operatorname{Im}\left(X_{i}\right)=0$; then $X_{i}=\operatorname{Re}\left(X_{i}\right)$. According to the relationship $Y_{i}=K_{i} X_{i}$, the output matrix $Y_{i}$ has no imaginary part; that is, $\operatorname{Im}\left(Y_{i}\right)=0$; then $Y_{i}=\operatorname{Re}\left(Y_{i}\right)$. Substituting $r=\cos \theta+j \sin \theta$ into $M_{i}$ gives

$$
\begin{aligned}
M_{i}= & \cos \theta A_{i i} X_{i}+\cos \theta X_{i} A_{i i}^{T}+\cos \theta A_{i j} X_{i}+\cos \theta X_{i} A_{i j}^{T} \\
& +\alpha_{i} D_{a i i} D_{a i i}^{T}+\sum_{j=1, j \neq i}^{N} \gamma_{i j} D_{a i j} D_{a i j}^{T}+\beta_{i} B_{i} D_{b i} D_{b i}^{T} B_{i}^{T} \\
& +\cos \theta B_{i} Y_{i}+\cos \theta Y_{i}^{T} B_{i}^{T} \\
& +j\left(\sin \theta A_{i i} X_{i}-\sin \theta X_{i} A_{i i}^{T}+\sin \theta A_{i j} X_{i}-\sin \theta X_{i} A_{i j}^{T}\right. \\
& \left.\quad+\sin \theta B_{i} Y_{i}-\sin \theta Y_{i}^{T} B_{i}^{T}\right) .
\end{aligned}
$$

Based on Lemma 7, the complex LMI (18) is transformed into the real LMI. Consider

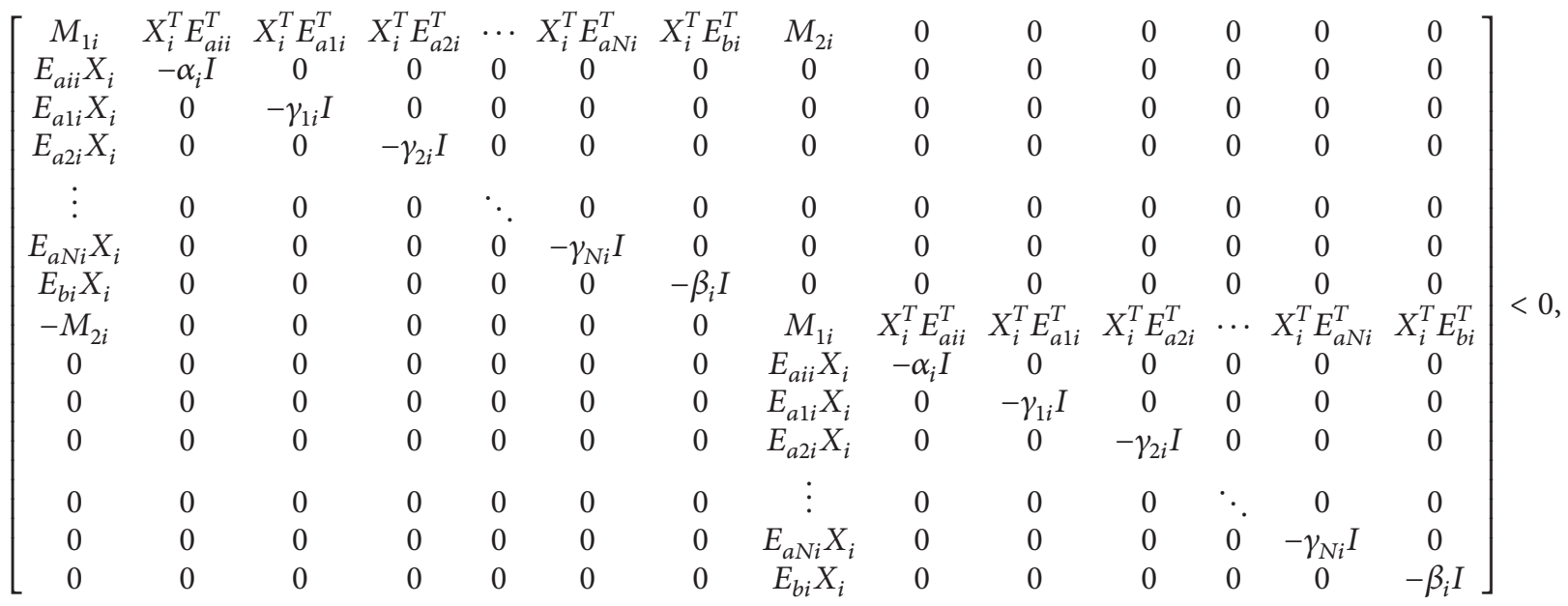

where $M_{1 i}=\operatorname{Re}\left(M_{i}\right)$ and $M_{2 i}=\operatorname{Im}\left(M_{i}\right)$. This completes the proof.

Therefore, the sufficient condition for decentralized robust stabilization of fractional order large-scale interconnected system with norm-bounded uncertainties under additive gain perturbations is derived. Furthermore, this condition is transformed into the solvability problem of linear matrix inequalities. In summary, by solving the LMI (18), we derive the sufficient condition on stabilizability via decentralized state feedback of the fractional order uncertain system with order $1<\alpha<2$.

\section{Nonfragile Controller Design of Fractional Order Large-Scale System with Multiplicative Gain Perturbations}

In this section, the nonfragile controller design problem is formulated for the fractional order large-scale interconnected system under multiplicative gain perturbations. Sufficient conditions are established for the decentralized stabilization of fractional order large-scale interconnected system with norm-bounded uncertainties under multiplicative gain perturbations. We are in a position to present our main result.

Theorem 9. Consider the fractional order large-scale uncertain system (1) with a commensurate order $1<\alpha<2$. Let 
$r=e^{j(\alpha-1)(\pi / 2)}$. The fractional order large-scale uncertain system can be stabilized via decentralized state feedback $u_{i}(t)=$ $\left(K_{i}+\Delta K_{i}\right) x_{i}(t)$ if there exist positive-definite block diagonal matrices $P_{i}=$ block $\operatorname{diag}\left[P_{1}, P_{2} \ldots, P_{N}\right]$, matrix $Y_{i}$ and positive number $\alpha_{i}, \gamma_{i j}, \beta_{i}, i, j=1,2, \ldots, N$, such that the following matrix inequalities hold:

$$
\left[\begin{array}{cccccccccccccc}
M_{1 i} & X_{i}^{T} E_{a i i}^{T} & X_{i}^{T} E_{a 1 i}^{T} & X_{i}^{T} E_{a 2 i}^{T} & \cdots & X_{i}^{T} E_{a N i}^{T} & Y_{i}^{T} E_{d i}^{T} & M_{2 i} & 0 & 0 & 0 & 0 & 0 & 0 \\
E_{a i i} X_{i} & -\alpha_{i} I & 0 & 0 & 0 & 0 & 0 & 0 & 0 & 0 & 0 & 0 & 0 & 0 \\
E_{a 1 i} X_{i} & 0 & -\gamma_{1 i} I & 0 & 0 & 0 & 0 & 0 & 0 & 0 & 0 & 0 & 0 & 0 \\
E_{a 2 i} X_{i} & 0 & 0 & -\gamma_{2 i} I & 0 & 0 & 0 & 0 & 0 & 0 & 0 & 0 & 0 & 0 \\
\vdots & 0 & 0 & 0 & \ddots & 0 & 0 & 0 & 0 & 0 & 0 & 0 & 0 & 0 \\
E_{a N i} X_{i} & 0 & 0 & 0 & 0 & -\gamma_{N i} I & 0 & 0 & 0 & 0 & 0 & 0 & 0 & 0 \\
E_{d i} Y_{i} & 0 & 0 & 0 & 0 & 0 & -\beta_{i} I & 0 & 0 & 0 & 0 & 0 & 0 & 0 \\
-M_{2 i} & 0 & 0 & 0 & 0 & 0 & 0 & M_{1 i} & X_{i}^{T} E_{a i i}^{T} & X_{i}^{T} E_{a 1 i}^{T} & X_{i}^{T} E_{a 2 i}^{T} & \cdots & X_{i}^{T} E_{a N i}^{T} & Y_{i}^{T} E_{d i}^{T} \\
0 & 0 & 0 & 0 & 0 & 0 & 0 & E_{a i i} X_{i} & -\alpha_{i} I & 0 & 0 & 0 & 0 & 0 \\
0 & 0 & 0 & 0 & 0 & 0 & 0 & E_{a 1 i} X_{i} & 0 & -\gamma_{1 i} I & 0 & 0 & 0 & 0 \\
0 & 0 & 0 & 0 & 0 & 0 & 0 & E_{a 2 i} X_{i} & 0 & 0 & -\gamma_{2 i} I & 0 & 0 & 0 \\
0 & 0 & 0 & 0 & 0 & 0 & 0 & \vdots & 0 & 0 & 0 & \ddots & 0 & 0 \\
0 & 0 & 0 & 0 & 0 & 0 & 0 & E_{a N i} X_{i} & 0 & 0 & 0 & 0 & -\gamma_{N i} I & 0 \\
0 & 0 & 0 & 0 & 0 & 0 & 0 & E_{d i} Y_{i} & 0 & 0 & 0 & 0 & 0 & -\beta_{i} I
\end{array}\right]
$$

where $M_{i}=r P_{i} A_{i i}+\bar{r} A_{i i}^{T} P_{i}+r P_{i} A_{i j}+\bar{r} A_{i j}^{T} P_{i}+\alpha_{i} D_{a i i} D_{a i i}^{T}+$ $\sum_{j=1, j}^{N} \gamma_{i j} D_{a i j} D_{a i j}^{T}+\beta_{i} B_{i} D_{d i} D_{d i}^{T} B_{i}^{T}+r B_{i} Y_{i}+\bar{r} Y_{i}^{T} B_{i}^{T}, X_{i}=$ $P_{i}^{-1}$, and $M_{1 i}$ and $M_{2 i}$ are the real part and imaginary part of matrices $M_{i}$, respectively. Moreover, the stabilization decentralized state-feedback gain matrix is given by $K_{i}=Y_{i} P_{i}$.

Proof. By the means of decentralized state-feedback control law (2), the closed-loop fractional order large-scale interconnected system is obtained as

$$
\begin{aligned}
\frac{d^{\alpha} x_{i}(t)}{d t^{\alpha}}= & {\left[A_{i i}+\Delta A_{i i}\right] x_{i}(t)+\sum_{j=1, j \neq i}^{N}\left[A_{i j}+\Delta A_{i j}\right] x_{j}(t) } \\
& +B_{i}\left[K_{i}+\Delta K_{i}\right] x_{i}(t),
\end{aligned}
$$

where $i, j=1,2, \ldots N, \Delta K_{i}=D_{b i} F_{b i} E_{b i} K_{i}$. Based on Lemma 6 , the necessary and sufficient condition on the asymptotical stability of the fractional order system with order $1<\alpha<2$ is that $r P A+\bar{r} A^{T} P<0$.

According to Definition 1, the sufficient condition on the stabilization of fractional order large-scale interconnected system satisfies the following quadratic matrix inequality:

$$
\begin{aligned}
& \sum_{i=1}^{N} \zeta^{*}\{r P[(A+\Delta A)+B(K+\Delta K)] \\
& \left.+\bar{r}[(A+\Delta A)+B(K+\Delta K)]^{T} P\right\} \zeta<0, \\
& \sum_{i=1}^{N}\left\{\zeta _ { i } ^ { * } \left[r P_{i} A_{i i}+\bar{r} A_{i i}^{T} P_{i}+r P_{i} \Delta A_{i i}+\bar{r} \Delta A_{i i}^{T} P_{i}\right.\right.
\end{aligned}
$$

$$
\begin{aligned}
& \left.+r P_{i} B_{i} K_{i}+\bar{r} K_{i}^{T} B_{i}^{T} P_{i}+r P_{i} B_{i} \Delta K_{i}+\bar{r} P_{i} \Delta K_{i}^{T} B_{i}^{T}\right] \zeta_{i} \\
+ & \left.2 r \zeta_{i}^{*}\left[\sum_{j=1, j \neq i}^{N}\left(A_{i j}+\Delta A_{i j}\right)\right] \zeta_{j}\right\}<0 .
\end{aligned}
$$

Consequently, the sufficient condition on the decentralized stabilization of fractional order large-scale interconnected system is that quadratic matrix inequality (23) holds.

Based on Lemmas 3 and 4, by means of enlarging the inequality we have

$$
r P_{i} \Delta A_{i i}+\bar{r} \Delta A_{i i}^{T} P_{i} \leq \alpha_{i} P_{i} D_{a i i} D_{a i i}^{T} P_{i}+\alpha_{i}^{-1} E_{a i i}^{T} E_{a i i} .
$$

Likewise, it is obtained that

$$
\begin{gathered}
r P_{i} B_{i} \Delta K_{i}+\bar{r} \Delta K_{i}^{T} B_{i}^{T} P_{i} \\
=r P_{i} B_{i} D_{d i} F_{d i} E_{d i} K_{i}+\bar{r} K_{i}^{T} E_{d i}^{T} F_{d i}^{T} D_{d i}^{T} B_{i}^{T} P_{i} \\
\leq \beta_{i} P_{i} B_{i} D_{d i} D_{d i}^{T} B_{i}^{T} P_{i}+\beta_{i}^{-1} K_{i}^{T} E_{d i}^{T} E_{d i} K_{i} \\
\sum_{i=1}^{N} \sum_{j=1, j \neq i}^{N} \zeta_{i}^{*}\left[r P_{i} \Delta A_{i j}+\bar{r} \Delta A_{i j}^{T} P_{i}\right] \zeta_{j} \\
\leq \sum_{i=1}^{N} \sum_{j=1, j \neq i}^{N} \zeta_{i}^{*} \gamma_{i j} P_{i} D_{a i j} D_{a i j}^{T} P_{i} \zeta_{i} \\
\quad+\sum_{j=1, j \neq i i=1}^{N} \sum_{j}^{N} \zeta_{j}^{*} \gamma_{i j}^{-1} E_{a i j}^{T} E_{a i j} \zeta_{j}
\end{gathered}
$$




$$
\begin{aligned}
\leq & \sum_{i=1}^{N} \sum_{j=1, j \neq i}^{N} \zeta_{i}^{*} \gamma_{i j} P_{i} D_{a i j} D_{a i j}^{T} P_{i} \zeta_{i} \\
& +\sum_{j=1, j \neq i i=1}^{N} \sum_{i}^{N} \zeta_{i}^{*} \gamma_{j i}^{-1} E_{a j i}^{T} E_{a j i} \zeta_{i} .
\end{aligned}
$$

Substituting (24), (25), and (26) into (24) results in the following quadratic matrix inequality, and we have

$$
\begin{aligned}
& \sum_{i=1}^{N}\left\{\zeta _ { i } ^ { * } \left[r P_{i} A_{i i}+\bar{r} A_{i i}^{T} P_{i}+r P_{i} A_{i j}+\bar{r} A_{i j}^{T} P_{i}\right.\right. \\
& +r P_{i} B_{i} K_{i}+\bar{r} K_{i}^{T} B_{i}^{T} P_{i}+\alpha_{i} P_{i} D_{a i i} D_{a i i}^{T} P_{i} \\
& +\sum_{j=1, j \neq i}^{N} \gamma_{i j} P_{i} D_{a i j} D_{a i j}^{T} P_{i}+\alpha_{i}^{-1} E_{a i i}^{T} E_{a i i} \\
& +\sum_{j=1, j \neq i}^{N} \gamma_{i j}^{-1} E_{a i j}^{T} E_{a i j}+\beta_{i}^{-1} K_{i}^{T} E_{b i}^{T} E_{b i} K_{i} \\
& \left.\left.+\beta_{i} P_{i} B_{i} D_{b i} D_{b i}^{T} B_{i}^{T} P_{i}\right] \zeta_{i}\right\}<0 \\
& \sum_{i=1}^{N}\left\{r P_{i} A_{i i}+\bar{r} A_{i i}^{T} P_{i}+r P_{i} A_{i j}+\bar{r} A_{i j}^{T} P_{i}\right. \\
& +r P_{i} B_{i} K_{i}+\bar{r} K_{i}^{T} B_{i}^{T} P_{i} \\
& +\alpha_{i} P_{i} D_{a i i} D_{a i i}^{T} P_{i}+P_{i} \sum_{j=1, j \neq i}^{N}\left(\gamma_{i j} D_{a i j} D_{a i j}^{T}\right) P_{i} \\
& +\alpha_{i}^{-1} E_{a i i}^{T} E_{a i i}+\sum_{j=1, j \neq i}^{N} \gamma_{i j}^{-1} E_{a i j}^{T} E_{a i j} \\
& \left.+\beta_{i} P_{i} B_{i} D_{d i} D_{d i}^{T} B_{i}^{T} P_{i}+\beta_{i}^{-1} K_{i}^{T} E_{d i}^{T} E_{d i} K_{i}\right\}<0 .
\end{aligned}
$$

Let $X_{i}=P_{i}^{-1}$ and $Y_{i}=K_{i} X_{i}$. By premultiplying and postmultiplying $P_{i}^{-1}$ onto (27), one has

$$
\begin{aligned}
& \sum_{i=1}^{N}\left\{r A_{i i} P_{i}^{-1}+\bar{r} P_{i}^{-1} A_{i i}^{T}+r A_{i j} P_{i}^{-1}+\bar{r} P_{i}^{-1} A_{i j}^{T}\right. \\
& +r B_{i} K_{i} P_{i}^{-1}+\bar{r} P_{i}^{-1} K_{i}^{T} B_{i}^{T} \\
& +\alpha_{i} D_{a i i} D_{a i i}^{T}+\sum_{j=1, j \neq i}^{N} \gamma_{i j} D_{a i j} D_{a i j}^{T}
\end{aligned}
$$

$$
\begin{aligned}
& +\alpha_{i}^{-1} P_{i}^{-1} E_{a i i}^{T} E_{a i i} P_{i}^{-1} \\
& +\sum_{j=1, j \neq i}^{N} \gamma_{i j}^{-1} P_{i}^{-1} E_{a i j}^{T} E_{a i j} P_{i}^{-1}+\beta_{i} B_{i} D_{d i} D_{d i}^{T} B_{i}^{T} \\
& \left.+\beta_{i}^{-1} P_{i}^{-1} K_{i}^{T} E_{d i}^{T} E_{d i} K_{i} P_{i}^{-1}\right\} \\
& =\sum_{i=1}^{N}\left\{r A_{i i} X_{i}+\bar{r} X_{i} A_{i i}^{T}+r A_{i j} X_{i}\right. \\
& +\bar{r} X_{i} A_{i j}^{T}+r B_{i} Y_{i}+\bar{r} Y_{i}^{T} B_{i}^{T} \\
& +\alpha_{i} D_{a i i} D_{a i i}^{T}+\sum_{j=1, j \neq i}^{N} \gamma_{i j} D_{a i j} D_{a i j}^{T} \\
& +\alpha_{i}^{-1} X_{i} E_{a i i}^{T} E_{a i i} X_{i} \\
& +\sum_{j=1, j \neq i}^{N} \gamma_{i j}^{-1} X_{i} E_{a i j}^{T} E_{a i j} X_{i} \\
& \left.+\beta_{i} B_{i} D_{d i} D_{d i}^{T} B_{i}^{T}+\beta_{i}^{-1} Y_{i}^{T} E_{d i}^{T} E_{d i} Y_{i}\right\}>0
\end{aligned}
$$

If the quadratic matrix inequality holds, then the fractional order large-scale interconnected system is asymptotically stable. By applying Schur complement, the above matrix inequality is equivalent to the following complex LMI:

$$
\left[\begin{array}{ccccccc}
M_{i} & X_{i}^{T} E_{a i i}^{T} & X_{i}^{T} E_{a 1 i}^{T} & X_{i}^{T} E_{a 2 i}^{T} & \cdots & X_{i}^{T} E_{a N i}^{T} & Y_{i}^{T} E_{d i}^{T} \\
E_{a i i} X_{i} & -\alpha_{i} I & 0 & 0 & 0 & 0 & 0 \\
E_{a 1 i} X_{i} & 0 & -\gamma_{1 i} I & 0 & 0 & 0 & 0 \\
E_{a 2 i} X_{i} & 0 & 0 & -\gamma_{2 i} I & 0 & 0 & 0 \\
\vdots & 0 & 0 & 0 & \ddots & 0 & 0 \\
E_{a N i} X_{i} & 0 & 0 & 0 & 0 & -\gamma_{N i} I & 0 \\
E_{d i} Y_{i} & 0 & 0 & 0 & 0 & 0 & -\beta_{i} I
\end{array}\right]
$$

$<0$,

where $M_{i}=r P_{i} A_{i i}+\bar{r} A_{i i}^{T} P_{i}+r P_{i} A_{i j}+\bar{r} A_{i j}^{T} P_{i}+\alpha_{i} D_{a i i} D_{a i i}^{T}+$ $\sum_{j=1, j \neq i}^{N} \gamma_{i j} D_{a i j} D_{a i j}^{T}+\beta_{i} B_{i} D_{d i} D_{d i}^{T} B_{i}^{T}+r B_{i} Y_{i}+\bar{r} Y_{i}^{T} B_{i}^{T}$. In practice, the feedback matrix $K_{i}$ has no imaginary part. So let $\operatorname{Im}\left(X_{i}\right)=0$; then $X_{i}=\operatorname{Re}\left(X_{i}\right)$. According to the relationship $Y_{i}=K_{i} X_{i}$, the output matrix $Y_{i}$ has no imaginary part; that is, 
$\operatorname{Im}\left(Y_{i}\right)=0$; then $Y_{i}=\operatorname{Re}\left(Y_{i}\right)$. Substituting $r=\cos \theta+j \sin \theta$ into $M_{i}$ gives

$$
\begin{aligned}
M_{i}= & \cos \theta A_{i i} X_{i}+\cos \theta X_{i} A_{i i}^{T}+\cos \theta A_{i j} X_{i}+\cos \theta X_{i} A_{i j}^{T} \\
& +\alpha_{i} D_{a i i} D_{a i i}^{T}+\sum_{j=1, j \neq i}^{N} \gamma_{i j} D_{a i j} D_{a i j}^{T}+\beta_{i} B_{i} D_{d i} D_{d i}^{T} B_{i}^{T} \\
& +\cos \theta B_{i} Y_{i}+\cos \theta Y_{i}^{T} B_{i}^{T}
\end{aligned}
$$

$$
\begin{aligned}
& +j\left(\sin \theta A_{i i} X_{i}-\sin \theta X_{i} A_{i i}^{T}+\sin \theta A_{i j} X_{i}-\sin \theta X_{i} A_{i j}^{T}\right. \\
& \left.\quad+\sin \theta B_{i} Y_{i}-\sin \theta Y_{i}^{T} B_{i}^{T}\right) .
\end{aligned}
$$

Based on Lemma 7, the complex LMI (30) is transformed into the real LMI. Consider

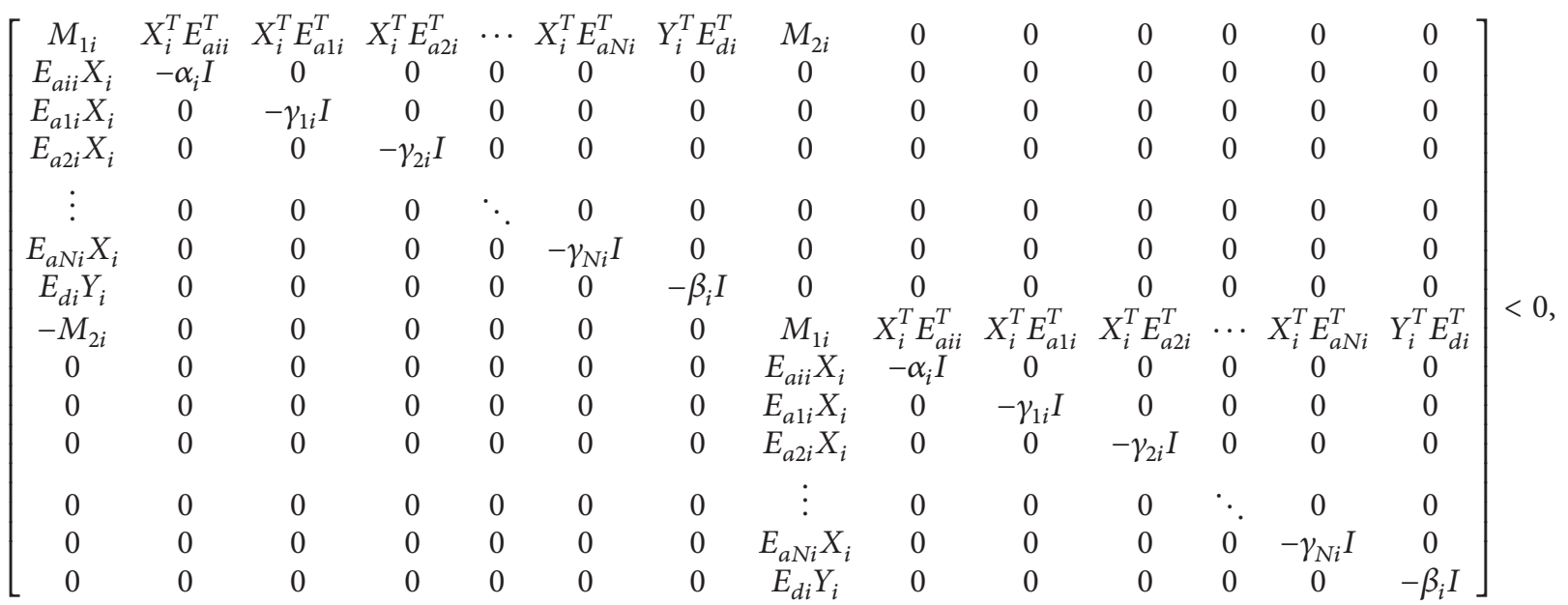

where $M_{1 i}=\operatorname{Re}\left(M_{i}\right), M_{2 i}=\operatorname{Im}\left(M_{i}\right)$.

This completes the proof.

Therefore, the sufficient condition for decentralized robust stabilization of fractional order large-scale interconnected system with norm-bounded uncertainties under multiplicative gain perturbations is obtained. Furthermore, this condition is transformed into the solvability problem of linear matrix inequalities. In summary, by solving the LMI (27), we derive the sufficient conditions on stabilizability via decentralized state feedback of the uncertain fractional order system under multiplicative gain perturbations.

\section{Numerical Examples}

In this section, to verify and demonstrate the effectiveness of the proposed method, two numerical examples are investigated. The fractional order large-scale interconnected uncertain system under controller gain perturbations is stabilized by the decentralized state feedback controllers. The Adams-type predictor-corrector method [29] is used for the numerical solution of fractional differential equations during the simulation.

Example 1. Consider the stabilization problem of fractional order large-scale interconnected uncertain system under additive gain perturbations:

$$
\begin{aligned}
\frac{d^{\alpha} x(t)}{d t^{\alpha}}= & {\left[A_{i i}+\Delta A_{i i}\right] x_{i}(t)+\sum_{j=1, j \neq i}^{N}\left[A_{i j}+\Delta A_{i j}\right] x_{j}(t) } \\
& +B_{i}\left[K_{i}+\Delta K_{i}\right] x_{i}(t)
\end{aligned}
$$

where $i, j=1,2, \ldots N, \alpha=1.5, N=2, \phi=\pi / 3$,

$$
\begin{aligned}
& A_{11}=\left[\begin{array}{cc}
-2.5 & 3.7 \\
1.9 & -2
\end{array}\right], \quad A_{12}=\left[\begin{array}{ll}
3.5 & 1.2 \\
1.2 & 3.5
\end{array}\right], \\
& A_{21}=\left[\begin{array}{cc}
0.2 & -0.1 \\
0.3 & 0.1
\end{array}\right], \quad A_{22}=\left[\begin{array}{cc}
1.8 & 1.2 \\
-1.2 & 0.8
\end{array}\right] \text {, } \\
& B_{1}=\left[\begin{array}{l}
1 \\
1
\end{array}\right], \quad B_{2}=\left[\begin{array}{cc}
0 & 15 \\
15 & 25
\end{array}\right] \\
& \Delta A_{11}=D_{a 11} F_{a 11} E_{a 11} \\
& =\left[\begin{array}{cc}
0.4 & 0.2 \\
1 & 0.3
\end{array}\right]\left[\begin{array}{cc}
\sin (\phi) & 0 \\
0 & \sin (\phi)
\end{array}\right]\left[\begin{array}{cc}
0.4 & 0.2 \\
1 & 0.3
\end{array}\right], \\
& \Delta A_{22}=D_{a 22} F_{a 22} E_{a 22} \\
& =\left[\begin{array}{cc}
0.4 & 0.2 \\
1 & 0.3
\end{array}\right]\left[\begin{array}{cc}
\sin (\phi) & 0 \\
0 & \sin (\phi)
\end{array}\right]\left[\begin{array}{ll}
0.3 & 0.5 \\
0.1 & 0.5
\end{array}\right],
\end{aligned}
$$




$$
\begin{aligned}
\Delta A_{12} & =D_{a 12} F_{a 12} E_{a 12} \\
& =\left[\begin{array}{l}
0.5 \\
0.3
\end{array}\right]\left[\begin{array}{cc}
\sin (\phi) & 0 \\
0 & \sin (\phi)
\end{array}\right]\left[\begin{array}{ll}
0.3 & 0.5 \\
0.1 & 0.5
\end{array}\right], \\
\Delta A_{21} & =D_{a 21} F_{a 21} E_{a 21} \\
& =\left[\begin{array}{c}
0.5 \\
0.3
\end{array}\right]\left[\begin{array}{cc}
\sin (\phi) & 0 \\
0 & \sin (\phi)
\end{array}\right]\left[\begin{array}{cc}
0.4 & 0.2 \\
1 & 0.3
\end{array}\right] .
\end{aligned}
$$

Meanwhile, the following additive gain perturbations are considered:

$$
\begin{aligned}
\Delta K_{1} & =D_{b 1} F_{b 1} E_{b 1}=\left[\begin{array}{ll}
0.5 & 0.3
\end{array}\right]\left[\begin{array}{cc}
\sin (\phi) & 0 \\
0 & \sin (\phi)
\end{array}\right]\left[\begin{array}{cc}
0.4 & 0.2 \\
1 & 0.3
\end{array}\right], \\
\Delta K_{2} & =D_{b 2} F_{b 2} E_{b 2}=\left[\begin{array}{cc}
0.5 & 0 \\
0 & 0.5
\end{array}\right]\left[\begin{array}{cc}
\sin (\phi) & 0 \\
0 & \sin (\phi)
\end{array}\right]\left[\begin{array}{cc}
0.5 & 0 \\
0 & 0.5
\end{array}\right] .
\end{aligned}
$$

By using the LMI technique, it is verified that the matrix inequalities are feasible in view of Theorem 8 . So the decentralized local state feedback gain matrix is obtained as

$$
\begin{aligned}
K_{1} & =\left[\begin{array}{ll}
-58.6565 & -61.7286
\end{array}\right], \\
K_{2} & =\left[\begin{array}{ll}
-0.2560 & -6.2675 \\
-1.7459 & -4.9510
\end{array}\right] .
\end{aligned}
$$

The time responses of system are shown in Figure 1. It is observed that its four states all converge to zero. It can be concluded that fractional order large-scale interconnected system with additive gain perturbations can be stabilized by the nonfragile controller.

Example 2. Consider the stabilization problem of fractional order large-scale interconnected uncertain system with multiplicative gain perturbations:

$$
\begin{aligned}
\frac{d^{\alpha} x(t)}{d t^{\alpha}}= & {\left[A_{i i}+\Delta A_{i i}\right] x_{i}(t)+\sum_{j=1, j \neq i}^{N}\left[A_{i j}+\Delta A_{i j}\right] x_{j}(t) } \\
& +B_{i}\left[K_{i}+\Delta K_{i}\right] x_{i}(t),
\end{aligned}
$$

where $i, j=1,2, \ldots, N, \alpha=1.5, N=2, \phi=\pi / 3$,

$$
\begin{aligned}
A_{11} & =\left[\begin{array}{cc}
-3.7 & 3.5 \\
1.9 & -3
\end{array}\right], \quad A_{12}=\left[\begin{array}{ll}
0.5 & 0.2 \\
0.2 & 0.5
\end{array}\right], \\
A_{21}= & {\left[\begin{array}{cc}
0.2 & -0.1 \\
0.3 & 0.1
\end{array}\right], \quad A_{22}=\left[\begin{array}{cc}
1 & 0.2 \\
-0.2 & 0.8
\end{array}\right], } \\
& B_{1}=\left[\begin{array}{c}
6.5 \\
0
\end{array}\right], \quad B_{2}=\left[\begin{array}{cc}
0.5 & 0.5 \\
0 & 1.5
\end{array}\right], \\
\Delta A_{11} & =D_{a 11} F_{a 11} E_{a 11} \\
& =\left[\begin{array}{cc}
0.4 & 0.2 \\
1 & 0.3
\end{array}\right]\left[\begin{array}{cc}
\sin (\phi) & 0 \\
0 & \sin (\phi)
\end{array}\right]\left[\begin{array}{cc}
0.4 & 0.2 \\
1 & 0.3
\end{array}\right],
\end{aligned}
$$

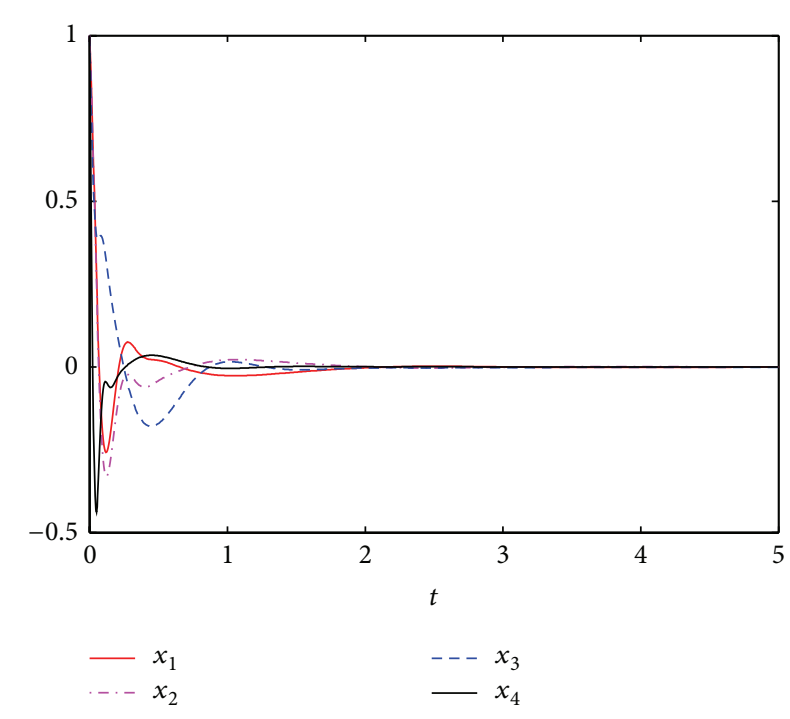

FIgURE 1: Example 1: time responses of the closed-loop fractional order large-scale interconnected uncertain system.

$$
\begin{aligned}
\Delta A_{22}= & D_{a 22} F_{a 22} E_{a 22} \\
= & {\left[\begin{array}{cc}
0.4 & 0.2 \\
1 & 0.3
\end{array}\right]\left[\begin{array}{cc}
\sin (\phi) & 0 \\
0 & \sin (\phi)
\end{array}\right]\left[\begin{array}{ll}
0.3 & 0.5 \\
0.1 & 0.5
\end{array}\right], } \\
\Delta A_{12} & =D_{a 12} F_{a 12} E_{a 12} \\
& =\left[\begin{array}{c}
0.5 \\
0.3
\end{array}\right]\left[\begin{array}{cc}
\sin (\phi) & 0 \\
0 & \sin (\phi)
\end{array}\right]\left[\begin{array}{ll}
0.3 & 0.5 \\
0.1 & 0.5
\end{array}\right], \\
\Delta A_{21} & =D_{a 21} F_{a 21} E_{a 21} \\
& =\left[\begin{array}{c}
0.5 \\
0.3
\end{array}\right]\left[\begin{array}{cc}
\sin (\phi) & 0 \\
0 & \sin (\phi)
\end{array}\right]\left[\begin{array}{cc}
0.4 & 0.2 \\
1 & 0.3
\end{array}\right] .
\end{aligned}
$$

Meanwhile, the following multiplicative gain perturbations are considered:

$$
\begin{array}{cc}
D_{d 1}=\left[\begin{array}{ll}
10.5 & 10.5
\end{array}\right], & E_{d 1}=\left[\begin{array}{l}
2.2 \\
2.2
\end{array}\right], \\
D_{d 2}=\left[\begin{array}{cc}
0.5 & 0 \\
0 & 0.5
\end{array}\right], & E_{d 2}=\left[\begin{array}{cc}
0.5 & 0 \\
0 & 0.5
\end{array}\right] .
\end{array}
$$

By using the LMI technique, it is verified that the matrix inequalities are feasible in view of Theorem 9. So the decentralized local state feedback gain matrix is obtained as

$$
K_{1}=\left[\begin{array}{ll}
-0.0304 & -0.0119
\end{array}\right], \quad K_{2}=\left[\begin{array}{cc}
-3.0473 & 2.8800 \\
2.0568 & -2.2810
\end{array}\right] .
$$

The time responses of system are shown in Figure 2. It is shown that its four states all converge to zero. It can be concluded that fractional order large-scale interconnected system with multiplicative gain perturbations can be stabilized by the nonfragile controller. 


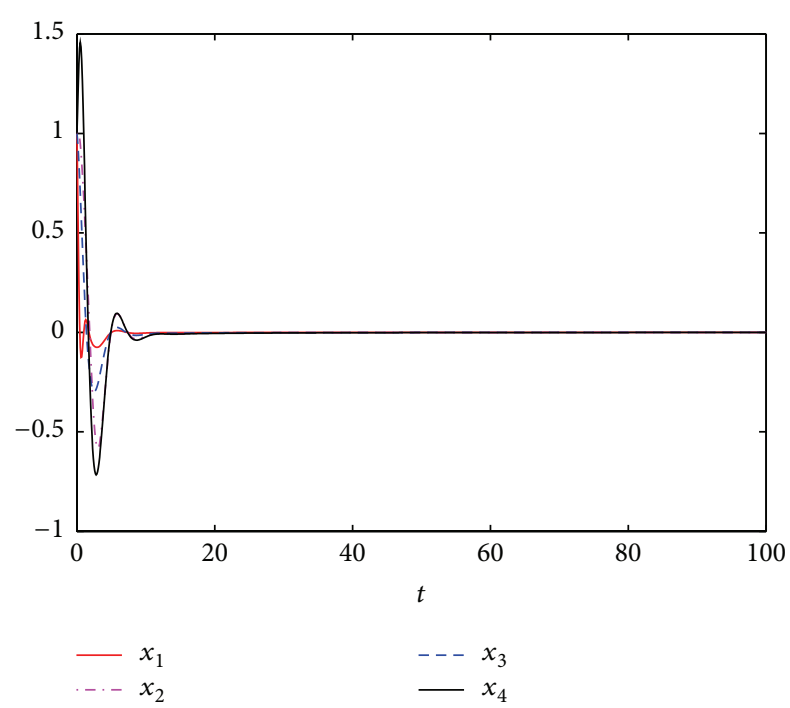

FIGURE 2: Example 2: time responses of the closed-loop fractional order large-scale interconnected uncertain system.

\section{Conclusions}

In this paper, sufficient conditions have been derived on the stabilization of fractional order large-scale interconnected uncertain system with a commensurate order $1<\alpha<2$ under two kinds of controller gain perturbations, that is, additive and multiplicative gain perturbations. The proposed method is based on the stability criterion of fractional order system by using the complex Lyapunov inequality. Moreover, the nonfragile controllers are designed. Simulation results have demonstrated the effectiveness of the proposed method.

\section{Conflict of Interests}

The author declares that there is no conflict of interests regarding to the publication of this paper.

\section{Acknowledgment}

This work was supported in part by a Zhiyuan Professorship at Shanghai Jiao Tong University.

\section{References}

[1] D.-Z. Zheng, "Decentralized output feedback stabilization of a class of nonlinear interconnected systems," IEEE Transactions on Automatic Control, vol. 34, no. 12, pp. 1297-1300, 1989.

[2] L. Bakule, "Decentralized control: an overview," Annual Reviews in Control, vol. 32, no. 1, pp. 87-98, 2008.

[3] J. Sandell, P. Varaiya, M. Athans, and M. G. Safonov, "Survey of decentralized control methods for large scale systems," IEEE Transactions on Automatic Control, vol. AC-23, no. 2, pp. 108$128,1978$.

[4] J. H. Chou, S. J. Ho, and I. R. Horng, "Robustness of disk-stability for perturbed large-scale systems," Automatica, vol. 28, no. 5, pp. 1063-1066, 1992.
[5] S. Ghosh, S. K. Das, and G. Ray, "Decentralized stabilization of uncertain systems with interconnection and feedback delays: an LMI approach," IEEE Transactions on Automatic Control, vol. 54, no. 4, pp. 905-912, 2009.

[6] J. H. Park, "Robust decentralized stabilization of uncertain large-scale discrete-time systems with delays," Journal of Optimization Theory and Applications, vol. 113, no. 1, pp. 105-119, 2002.

[7] O. M. Kown and J. H. Park, "Decentralized guaranteed cost control for uncertain large-scale systems using delayed feedback: LMI optimization approach," Journal of Optimization Theory and Applications, vol. 129, no. 3, pp. 391-414, 2006.

[8] G. J. Silva, A. Datta, and S. P. Bhattacharyya, PID Controllers for Time-Delay Systems, Birkhäuser, Boston, Mass, USA, 2004.

[9] L. H. Keel and S. P. Bhattacharyya, "Robust, fragile, or optimal?" IEEE Transactions on Automatic Control, vol. 42, no. 8, pp. 10981105, 1997.

[10] P. Dorato, "Non-fragile controller design: an overview," in Proceedings of the American Control Conference (ACC '98), vol. 5, pp. 2829-2831, June 1998.

[11] J. H. Park, "Robust non-fragile guaranteed cost control of uncertain large-scale systems with time-delays in subsystem interconnections," International Journal of Systems Science, vol. 35, no. 4, pp. 233-241, 2004.

[12] W. M. Haddad and J. R. Corrado, "Robust resilient dynamic controllers for systems with parametric uncertainty and controller gain variations," International Journal of Control, vol. 73, no. 15, pp. 1405-1423, 2000.

[13] H. Oya and K. Hagino, "Trajectory-based design of robust nonfragile controllers for a class of uncertain linear continuoustime systems," International Journal of Control, vol. 80, no. 12, pp. 1849-1862, 2007.

[14] Z. Feng and J. Lam, "Robust reliable dissipative filtering for discrete delay singular systems," Signal Processing, vol. 92, no. 12, pp. 3010-3025, 2012.

[15] Z. Feng and J. Lam, "Reliable dissipative control for singular Markovian systems," Asian Journal of Control, vol. 15, no. 3, pp. 901-910, 2013.

[16] D. Matignon, "Stability properties for generalized fractional differential systems," in Systèmes Différentiels Fractionnaires, Modèles, Méthodes et Applications, vol. 5 of ESAIM Proceedings, pp. 145-158, SMAI, Paris, France, 1998.

[17] I. Podlubny, Fractional Differential Equations, Academic Press, New York, NY, USA, 1999.

[18] C. Bonnet and J. R. Partington, "Coprime factorizations and stability of fractional differential systems," Systems \& Control Letters, vol. 41, no. 3, pp. 167-174, 2000.

[19] C. Bonnet and J. R. Partington, "Analysis of fractional delay systems of retarded and neutral type," Automatica, vol. 38, no. 7, pp. 1133-1138, 2002.

[20] C. Bonnet and J. R. Partington, "Stabilization of some fractional delay systems of neutral type," Automatica, vol. 43, no. 12, pp. 2047-2053, 2007.

[21] H.-S. Ahn, Y. Q. Chen, and I. Podlubny, "Robust stability test of a class of linear time-invariant interval fractional-order system using Lyapunov inequality," Applied Mathematics and Computation, vol. 187, no. 1, pp. 27-34, 2007.

[22] H. S. Ahn and Y. Chen, "Necessary and sufficient stability condition of fractional-order interval linear systems," Automatica, vol. 44, no. 11, pp. 2985-2988, 2008. 
[23] M. Moze, J. Sabatier, and A. Oustaloup, "LMI tools for stability analysis of fractional systems," in Proceedings of the ASME 2005 International Design Engineering Technical Conferences and Computers and Information in Engineering Conference (IDETC/CIE '05), pp. 1611-1619, Long Beach, Calif, USA, 2005.

[24] C. Farges, M. Moze, and J. Sabatier, "Pseudo-state feedback stabilization of commensurate fractional order systems," Automatica, vol. 46, no. 10, pp. 1730-1734, 2010.

[25] J. Y. Lin, "Robust resilient controllers synthesis for uncertain fractional-order large-scale interconnected system," Journal of the Franklin Institute. Engineering and Applied Mathematics, vol. 351, no. 3, pp. 1630-1643, 2014.

[26] R. A. Horn and C. R. Johnson, Matrix Analysis, Cambridge University Press, Cambridge, UK, 1985.

[27] P. P. Khargonekar, I. R. Petersen, and K. Zhou, "Robust stabilization of uncertain linear systems: quadratic stabilizability and $H_{\infty}$ control theory," IEEE Transactions on Automatic Control, vol. 35, no. 3, pp. 356-361, 1990.

[28] D. Matignon, "Stability results for fractional differential equations with applications to control processing," in Computational Engineering in Systems Applications, vol. 2, pp. 963-968, 1996.

[29] K. Diethelm, N. J. Ford, and A. D. Freed, "A predictor-corrector approach for the numerical solution of fractional differential equations," Nonlinear Dynamics, vol. 29, no. 1-4, pp. 3-22, 2002. 


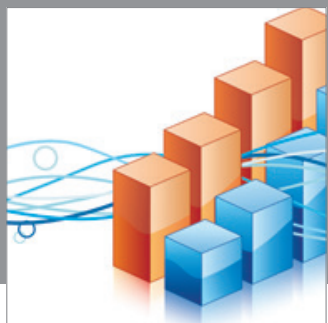

Advances in

Operations Research

mansans

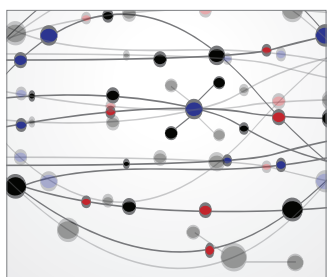

The Scientific World Journal
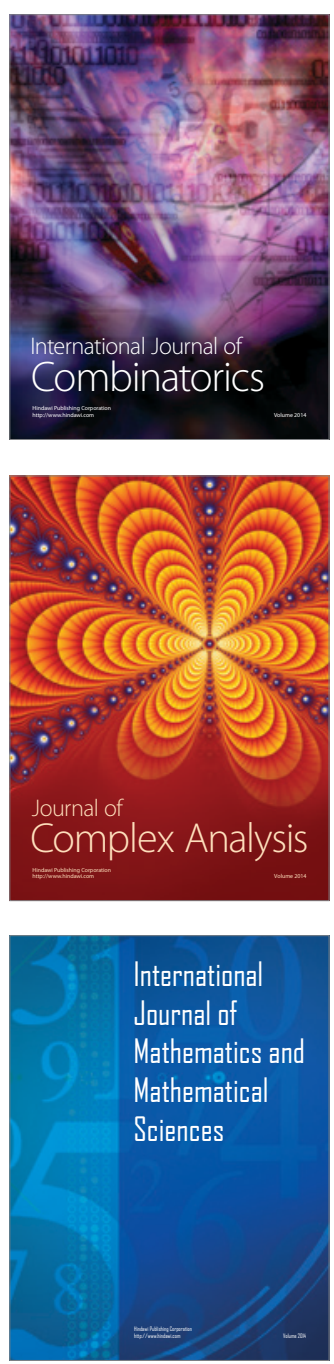
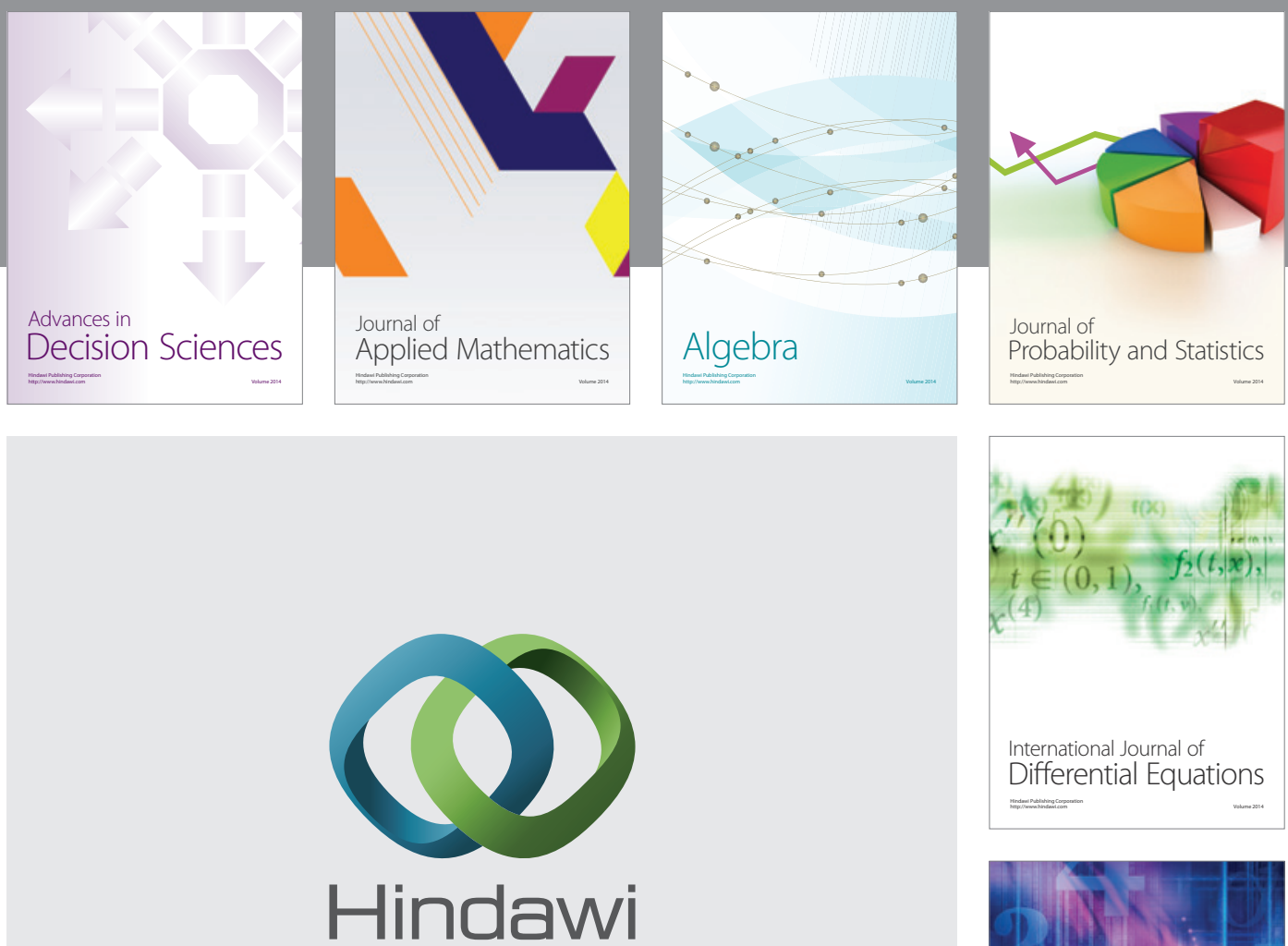

Submit your manuscripts at http://www.hindawi.com
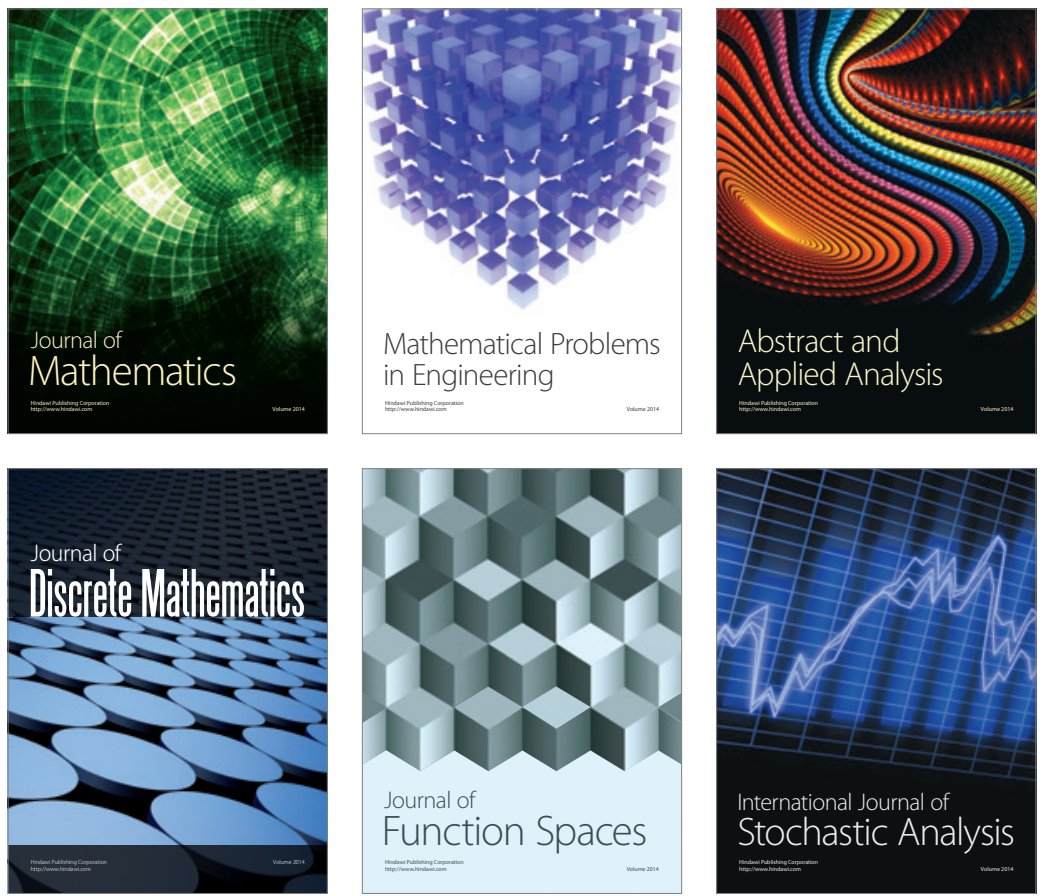

Journal of

Function Spaces

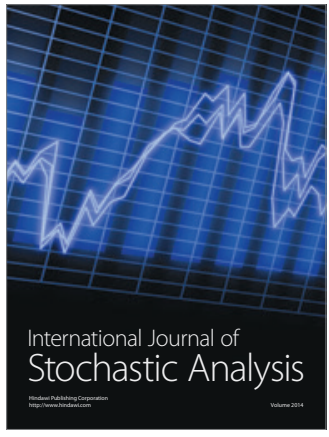

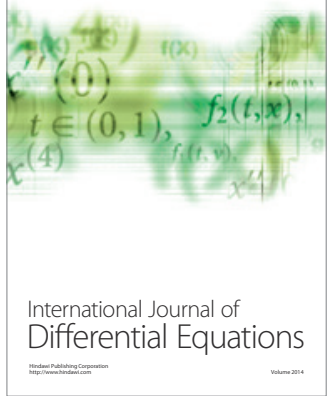
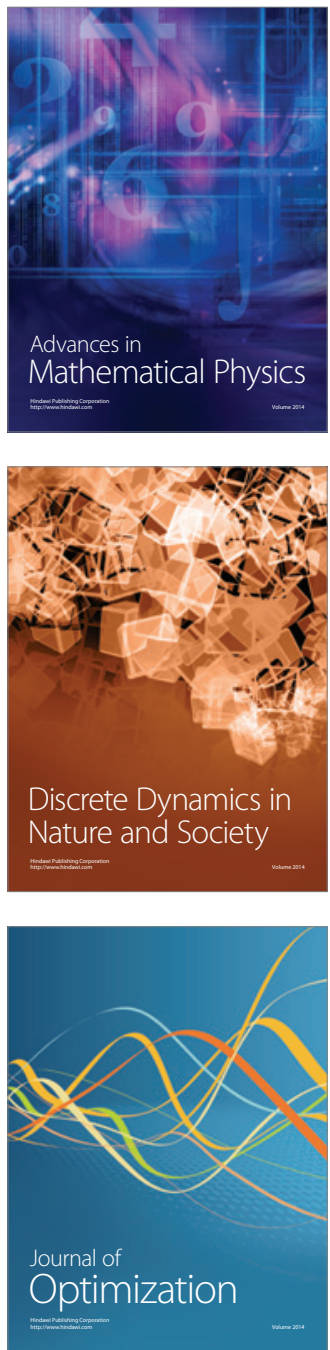